\title{
The fate of Krüppel-like factor 9-positive hepatic carcinoma cells may be determined by the programmed cell death protein 5
}

\author{
DA-ZHI FU, YING CHENG, HUI HE, HAI-YANG LIU and YONG-FENG LIU
}

Department of General Surgery, First Affiliated Hospital of China Medical University, Shenyang 110001, Liaoning, P.R. China

Received August 10, 2013; Accepted October 7, 2013

DOI: 10.3892/ijo.2013.2147

\begin{abstract}
Liver cancer in men is the fifth most frequently diagnosed cancer worldwide. Human Krüppel-like factor (KLF9) gene, localized on human chromosome 9q13, has been implicated in mediating a diverse range of biological processes including stem cell maintenance and differentiation of T- and B-lymphocytes. In this study, we confirmed that the levels of KLF9 mRNA and protein were lower in hepatocellular carcinoma (HCC) tissue compared to normal tissue. In addition, we confirmed that upregulation of KLF9 inhibited cell proliferation and mobility and induce apoptosis in HepG2 cells depending on programmed cell death protein 5 (PDCD5) expression. However, no interaction was found between KLF9 and PDCD5 using fluorescence resonance energy transfer (FRET) and co-immunoprecipitation (co-IP). We confirmed that PDCD5 overexpression stimulated the promoter activities of KLF9 by luciferase reporter assays.
\end{abstract}

\section{Introduction}

Liver cancer in men is the fifth most frequently diagnosed cancer worldwide but the second most frequent cause of cancer death (1). Half of these cases and deaths were estimated to occur in China (2).

Krüppel-like factor $(K L F)$ family members share a three $\mathrm{C} 2 \mathrm{H} 2$ zinc-finger DNA binding domain, and are involved in cell proliferation and differentiation control in normal as well as in pathological situations (3). An emerging body of evidence indicates that KLF is associated with various types of cancers. Downregulation of KLF4 expression is reported in esophageal cancer (4) and colorectal cancer (5). Significant genetic alterations of KLF6 expression are observed in prostate cancer (6). LOH of KLF6 locus at chromosome 10p15 contributes to the development of colorectal carcinoma (7).

Correspondence to: Dr Yong-Feng Liu, Department of General Surgery, First Affiliated Hospital of China Medical University, 155 Nanjing Road, Heping District, Shenyang 110001, Liaoning, P.R. China

E-mail: yfliu_2006@163.com

Key words: hepatocellular carcinoma, PDCD5, KLF9, apoptosis, fluorescence resonance energy transfer
In the study of Kang et al (8), downregulation of KLF9 is confirmed in human colorectal cancer. However, the roles of KLF9 in hepatocellular carcinoma (HCC) were not clear. $K L F 9$ is an evolutionary well-conserved member of the $K L F$ family of transcriptional regulators (9). KLF9 is first identified as a transcriptional repressor of the rat Cyplal (previously $P-4501 A 1)$ gene and originally named basic transcription element-binding protein 1 (Bteb1) (10). Human KLF9 gene, localized on human chromosome 9q13 (11), has been implicated in mediating a diverse range of biological processes including stem cell maintenance (12) and differentiation of T- and B-lymphocytes $(13,14)$.

In our previous study, we confirmed that the levels of PDCD5 mRNA and protein were lower in HCC tissue than normal tissue (15). In this study, we investigated the potential role of KLF9 in HCC. We first demonstrated that KLF9 expression is correlated with clinicopathological features and patient survival, and it may be a useful predictor of prognosis in patients with HCC. Interesting, our results also showed that exogenous KLF9 expression played its role in HepG2 cells depending on PDCD5 expression.

\section{Materials and methods}

Cell culture and tumor specimens. Human liver cancer cell line, HepG2, was cultured in DMEM (Hyclone, Logan, UT, USA) containing $10 \%$ fetal bovine serum (Invitrogen Gibco, Carlsbad, $\mathrm{CA}, \mathrm{USA}$ ) and incubated in a $5 \% \mathrm{CO}_{2}$ incubator at $37^{\circ} \mathrm{C}$. Liver specimens were derived from 56 patients undergoing surgical resection of primary hepatocellular carcinoma without prior chemotherapeutic treatment or radiotherapy. Basic information of these patients was described in our previous study (15).

Quantitative real-time $R T-P C R$ ( $Q R T-P C R)$. Total cellular RNA $(1 \mu \mathrm{g})$ from each cell line was reverse-transcribed using Takara Reverse Transcription kit (Takara, Dalian, China) and oligo(dT) 15 primers (Takara). The KLF9 primers were: 5'-TGGCTGTGGGAAAGTCTATGG-3' (sense) and 5'-CTC GTCTGAGCGGGAGAACT-3' (antisense). SYBR Green real-time PCR was performed with an ABI PRISM 7500 Sequence Detector. Thermal cycling conditions included preincubation at $50^{\circ} \mathrm{C}$ for $2 \mathrm{~min}, 95^{\circ} \mathrm{C}$ for $10 \mathrm{~min}$ followed by $40 \mathrm{PCR}$ cycles at $95^{\circ} \mathrm{C}$ for $15 \mathrm{sec}$ and $60^{\circ} \mathrm{C}$ for $1 \mathrm{~min}$. Relative transcript levels were calculated using the relative standard curve method and results were normalized to GAPDH. The 
GAPDH primers were: 5'-AGAAGGCTGGGGCTCATTTG-3' (sense) and 5'-AGGGGCCATCCACAGTCTTC-3' (antisense).

Immunohistochemical staining (IHC). As described in our previous study (15), 4- $\mu \mathrm{m}$ sections of paraffin-embedded specimens were performed using the KLF9 polyclonal antibody (sc-12996, Santa Cruz Biotechnology, Santa Cruz, CA, USA). Briefly, after deparaffinization and hydration, the endogenous peroxidase activity was quenched by a 30 -min incubation in a mixture of $0.3 \%$ hydrogen peroxide solution in $100 \%$ methanol. The sections were blocked for $2 \mathrm{~h}$ at room temperature with $1.5 \%$ blocking serum in PBS and incubated with KLF9 antibody (1:1,000 dilution) at $4^{\circ} \mathrm{C}$ in a moist chamber overnight, followed by incubation with Envision reagent (Dako, Carpinteria, CA, USA) and color development in 3,3'-diaminobenzidine tetrahydrochloride (DAB, Sigma-Aldrich, Carlsbad, CA, USA). The slides were then lightly counterstained with hematoxylin, dehydrated with ethanol, cleaned with xylene, and mounted. Adjacent non-cancer tissues were used as controls. Sections treated without primary antibodies were used as negative controls. The positive percentage of counting cells was graded semi-quantitatively according to a four-tier scoring system: negative (-), $0-5 \%$; weakly positive (+), $6-25 \%$; moderately positive (++), 26-50\%; and strongly positive (+++), 51-100\%.

Plasmid construction and transfection. The recombinant plasmid, pEGFP-C1-KLF9 was kindly gifted by Mr. Lin Niu (China Medical University) and verified by DNA sequencing (Sunbiotech, Beijing, China). pEGFP-C1-KLF9 transfections were performed using Lipofectamine 2000 (Invitrogen) according to the manufacturer's instructions.

Colony formation assay. Cells were seeded at 200 cells per well in 24-well tissue culture plates. Plates were incubated for one week in a humidified incubator at $37^{\circ} \mathrm{C}$. One week after seeding, colonies were stained with $0.05 \%$ crystal violet containing 50\% methanol and counted. The colonies were counted in 4-5 random fields for each of the duplicate samples by using a microscope at X100 magnification.

Transmission electron microscopy. Specimens were immersed in $2 \%$ cacodylate-buffered glutaraldehyde, rinsed in cacodylate buffer supplemented with $15 \%$ sucrose, post-fixed with $1 \%$ phosphate-buffered $\mathrm{OsO}_{4}$, dehydrated with alcohol, clarified in propylene oxide, and embedded in Epon using flat molds. Ultrathin sections were made with an ultramicrotome, stained with uranyl acetate, followed by a saturated solution of bismuth subnitrate, and observed under a JEOL JSM 6400 scanning electron microscope (JEOL, Tokyo, Japan).

Cell apoptosis assay. Cells $\left(5 \times 10^{5}\right)$ were collected without EDTA and washed with PBS. A 500- $\mu 1$ binding buffer, $5 \mu 1$ Annexin V-FITC and $5 \mu \mathrm{l}$ propidium iodide (PI) (KeyGen, Nanjing, China) were added into the suspension in that order and mixed at room temperature in the dark for $10 \mathrm{~min}$. The examination was performed by a FACSCalibur machine (BD Biosciences, Baltimore, MD, USA) within $1 \mathrm{~h}$.

Determination of mitochondrial membrane potential. The mitochondrial membrane potential (MMP) was analyzed using the fluorescent dye 5,5',6,6'-tetrachloro-1,1',3,3'-tetraethylbenzimidazolycarbocyanine iodide (JC-1) following the manufacturer's protocol (KeyGen). Briefly, cells were plated in a 6-well culture plate. After $24 \mathrm{~h}$, cells were washed twice with PBS, harvested and loaded with $20 \mathrm{nM} \mathrm{JC}-1$ for $30 \mathrm{~min}$ in the dark. Afterwards, MMP was analyzed by a FACSCalibur machine as described above.

In vitro wound healing assay. Cells were grown in a 6-well dish. A confluent monolayer of cells was scratched with a 200- $\mu$ l pipette tip to simulate a wound. Cells were washed twice with PBS and then supplemented with medium and incubated for $4 \mathrm{~h}$ at $37^{\circ} \mathrm{C}$. Cell migration into the wounded area was monitored microscopically. Images were captured at the interface of the unwounded and wounded areas.

Cell invasion assay. For invasive assay, cells were resuspended in serum-free DMEM, and seeded in the control-membrane insert on the top portion of the Matrigel-coated chamber (BD Bioscience). The lower compartment of the chamber contained $10 \%$ FBS as a chemo-attractant. After incubation for $24 \mathrm{~h}$, cells on the membrane were scrubbed, washed with PBS and fixed in $100 \%$ methanol and stained with Giemsa dye.

Imaging with confocal FRET microscopy. Confocal images were acquired with a Leica TCS-SP2 confocal microscope (Leica Microsystems, Heidelberg GmbH, Germany). To correct for spectral bleed-through (SBT) and for uncontrolled variations in donor-acceptor concentrations, a combination of donor, FRET and acceptor filter sets was used to isolate and maximize three specific signals: donor fluorescence, acceptor fluorescence resulting from FRET and the directly excited acceptor fluorescence, respectively. Filter sets used were as follows: the red channel (donor excitation/donor emission $=543 / 575 \mathrm{~nm}$ ), the green/blue channel (acceptor excitation/ acceptor emission $=633 / 680 \mathrm{~nm}$ ) and the FRET channel $($ donor excitation/acceptor emission $=543 / 680 \mathrm{~nm}$ : FRET $)$.

Co-immunoprecipitation. Transfected cells were washed once with PBS, lysed for $30 \mathrm{~min}$ in lysis buffer $(50 \mathrm{mM}$ Tris-HCl, pH 7.5, $150 \mathrm{mM} \mathrm{NaCl}, 1 \%$ Nonidet P-40) containing protease inhibitors (Cocktail, Roche, Basel, Switzerland) and phosphatase inhibitors $\left(1 \mathrm{mM} \mathrm{NaF}\right.$ and $\left.1 \mathrm{mM} \mathrm{Na}_{3} \mathrm{VO}_{4}\right)$, and centrifuged at $15,000 \mathrm{xg}$ at $4^{\circ} \mathrm{C}$ for $15 \mathrm{~min}$. Supernatants were pre-cleared with EZ View Red protein G-Sepharose (Sigma) for $1 \mathrm{~h}$ at $4^{\circ} \mathrm{C}$. Then $5 \mu \mathrm{g}$ of antibody specific for each target protein were added in each sample. Immune complexes were precipitated by EZ View red protein G-Sepharose overnight at $4^{\circ} \mathrm{C}$ and washed 3 times with lysis buffer. The immune complexes were boiled $\left(100^{\circ} \mathrm{C}\right)$ for $10 \mathrm{~min}$ in SDS sample buffer (100 mM Tris-HCl, pH 8.8, 0.01\% bromophenol blue, $20 \%$ glycerol, $4 \%$ SDS) containing $10 \mathrm{mM}$ dithiothreitol and resolved by $10 \%$ SDS-PAGE.

Luciferase assays. The methods of Du et al (16), were used for transfection with $0.4 \mu \mathrm{g}$ of either pGL3-control-692-bp KLF9 promoter or pGL3-control-131-bp KLF9 promoter in 24-well plates. Each group of cells was cotransfected with either $1.0 \mu \mathrm{g}$ of pcDNA3.1-PDCD5 vector or empty pcDNA3.1 vector. All cells were cotransfected with $10 \mathrm{ng}$ of pRL-TK to control for 

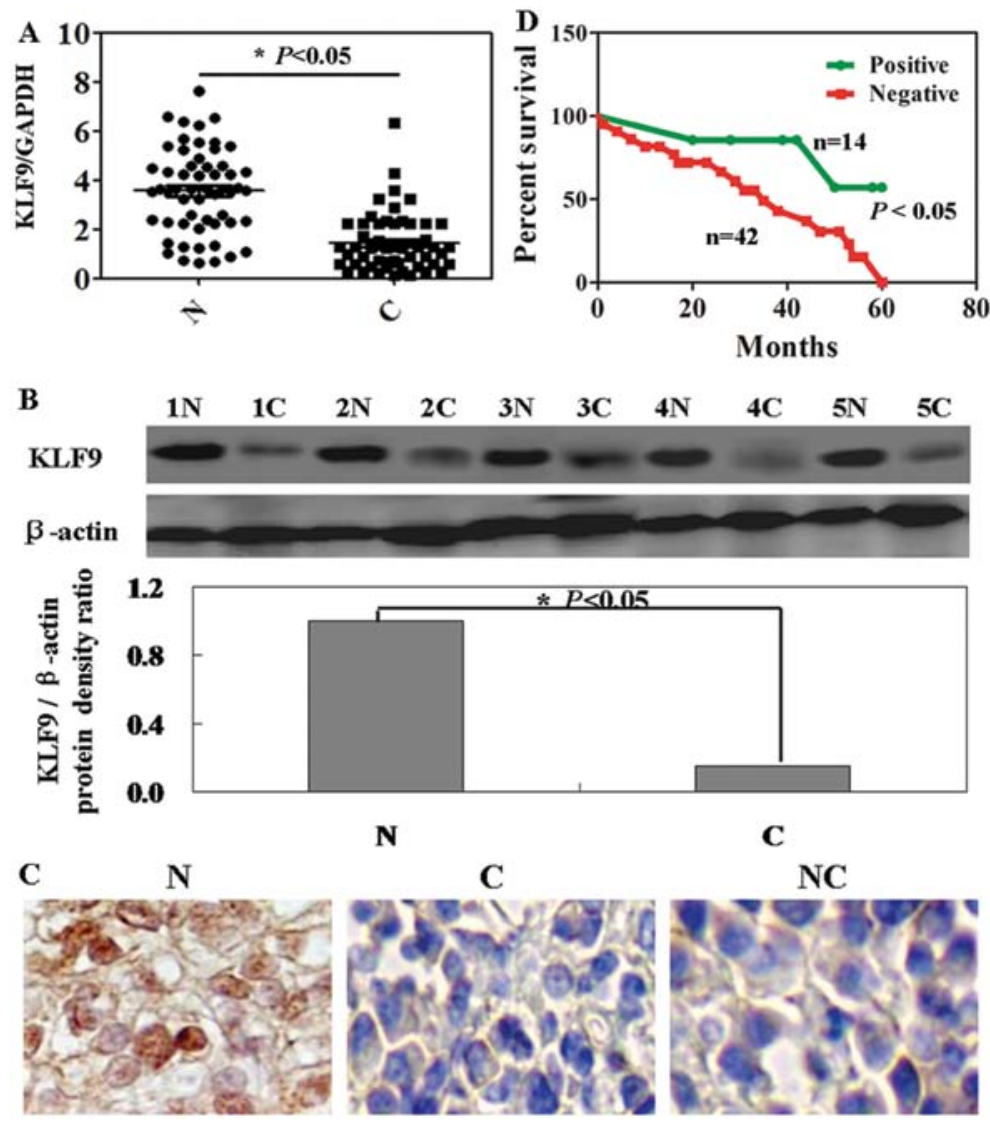

Figure 1. (A) The level of KLF9 mRNA was measured in specimens by real-time PCR. GADPH was the internal control. (B) Representative results of two paired of HCC and corresponding normal tissue by western blot analysis. $\beta$-actin was the internal control. (C) Immunohistochemical staining for KLF9 protein in specimens. The nuclei were counterstained with hematoxylin. (D) Kaplan-Meier curves for cumulative survival rate of the patients with HCC according to the KLF9 expression. N, normal; C, cancer; NC, negative control.

transfection efficiency. After $4 \mathrm{~h}$, the media were changed, and 48-h posttransfection, the cells were rinsed with cold PBS and lysed with 1X reporter lysis buffer (Promega, Madison, WI, USA). The lysate was collected after two freeze/thaw cycles. Luciferase activity was measured using the luciferase reagent kit (Promega). Transfections were performed in duplicate, and experiments were repeated three times. The pGL3-control vector was used as a control.

Western blot analysis. Whole protein was extracted from human liver cancer cell lines and liver tissues using RIPA buffer (20 mM Tris-HCl, $150 \mathrm{mM} \mathrm{NaCl}, 2 \mathrm{mM}$ EDTA, $1 \%$ Triton-X100) containing a protease inhibitor cocktail (Sigma). Extract protein amounts were quantified using the BCA protein assay kit (CWbiotech, Beijing, China). Equivalent amounts of protein $(60 \mu \mathrm{g})$ were separated using $10 \%$ SDS-PAGE and transferred to a PVDF membrane (Millipore Corp., Billerica, MA, USA). Western blot analysis was performed using primary antibodies: KLF9 (sc-376422) and $\beta$-actin (sc-130657, Santa Cruz Biotechnology). Each specific antibody binding was detected with horseradish peroxidase (HRP)-conjugated respective secondary antibodies (Amersham Biosciences, Amersham, UK) and ECL solutions (Amersham Biosciences).

Affymetrix GeneChip technology. The total RNA was extracted from cells as described above. The total RNA samples were then analyzed by CapitalBio Corp for GeneChip (Affymetrix) assay. Furthermore, each treatment has 3 biological replicates that were measured in this manner. Gene expression analysis was performed by using the Affymetrix (Santa Clara, CA, USA) GeneChip, following the laboratory methods in the Affymetrix GeneChip expression manual. Gene expression analysis was performed using triple arrays and triple independent mRNA samples for each treatment. Microarray data were analyzed by using Bio MAS 3.0 software (CapitalBio, Beijing, China). Using the criterion of cutoff limitation as a fold change $\geq 2$ or $\leq 0.5$ and $q$-value $\leq 5 \%$, differential expression genes were screened and clustered.

Statistical analysis. Statistical analyses were performed using SPSS 15.0 software (SPSS, Chicago, IL, USA). Comparisons were made using $\chi^{2}$ tests, the Wilcoxon signed-rank test and the t-test. Overall survival was analyzed using the KaplanMeier method and the significance of differences in survival rates was estimated using the log-rank test. P-values of $<0.05$ were considered significant.

\section{Results}

Reduction of KLF9 in HCC tissues compared with normal tissues. In hepatoma tumor tissues, downregulation of KLF9 mRNA and protein was detected in all 56 cases compared with each individual normal tissue (Fig. $1 \mathrm{~A}$ and $\mathrm{B} ; \mathrm{P}<0.05)$. In 
Table I. KLF9 expression associated with demographic and biological parameters in 56 hepatocellular carcinoma samples.

\begin{tabular}{|c|c|c|c|c|c|c|c|c|}
\hline \multirow[b]{2}{*}{ Clinicopathological features } & \multirow[b]{2}{*}{$\mathrm{n}$} & \multicolumn{7}{|c|}{ KLF9 expression } \\
\hline & & - & + & ++ & +++ & $\mathrm{PR}(\%)$ & $\chi^{2}$ & P-value \\
\hline Sex & & & & & & & 10.84 & 0.012 \\
\hline Female & 20 & 10 & 4 & 3 & 3 & 50.0 & & \\
\hline Male & 36 & 32 & 1 & 1 & 2 & 11.1 & & \\
\hline Age (years) & & & & & & & 5.72 & 0.126 \\
\hline$<55$ & 26 & 16 & 4 & 2 & 4 & 38.5 & & \\
\hline$\geq 55$ & 30 & 26 & 1 & 2 & 1 & 13.3 & & \\
\hline Tumor number & & & & & & & 4.09 & 0.252 \\
\hline Multiple & 38 & 30 & 3 & 1 & 4 & 21.1 & & \\
\hline Solitary & 18 & 12 & 2 & 3 & 1 & 33.3 & & \\
\hline Differentiation & & & & & & & 0.42 & 0.935 \\
\hline Differentiated & 27 & 20 & 2 & 2 & 3 & 25.9 & & \\
\hline Undifferentiated & 29 & 22 & 3 & 2 & 2 & 24.1 & & \\
\hline Portal invasion & & & & & & & 6.48 & 0.090 \\
\hline- & 17 & 11 & 1 & 1 & 4 & 35.3 & & \\
\hline+ & 39 & 31 & 4 & 3 & 1 & 20.5 & & \\
\hline Lymph node metastasis & & & & & & & 8.46 & 0.037 \\
\hline- & 18 & 10 & 3 & 1 & 4 & 44.4 & & \\
\hline+ & 38 & 32 & 2 & 3 & 1 & 15.8 & & \\
\hline Tumor size (cm) & & & & & & & 2.31 & 0.511 \\
\hline$<5$ & 25 & 17 & 3 & 3 & 2 & 32.0 & & \\
\hline$\geq 5$ & 31 & 25 & 2 & 1 & 3 & 19.4 & & \\
\hline HBV infection & & & & & & & 6.84 & 0.077 \\
\hline- & 22 & 13 & 2 & 3 & 4 & 40.9 & & \\
\hline+ & 34 & 29 & 3 & 1 & 1 & 14.7 & & \\
\hline
\end{tabular}

PR positive rate, $\chi^{2}$ value, $\chi^{2}$ distribution.

Fig. 1C, the results of immunohistochemical staining showed that positive staining was seen in the nucleus of the normal cells, in contrast, almost no positive cells were seen in cancer tissue. We failed to detect any significant association between KLF9 expression and age, tumor number, differentiation, portal invasion and HBV infection (Table I; P>0.05). However, the KLF9 expression was correlated statistically with sex $(\mathrm{P}=0.012)$ and lymph node metastasis $(\mathrm{P}=0.037)$. Cox's proportional hazard analysis indicated that KLF9 expression was an independent prognostic factor for HCC (Table II; $\mathrm{P}<0.05$ ). HCC patients with KLF9 expression were associated with a significantly higher survival rate than the ones without KLF9 expression (Fig. 1D; $\mathrm{P}=0.023$ ).

PDCD5 and KLF9-expressing HepG2 cell lines. We investigated the consequence of exogenous KLF9 and PDCD5 expression in HepG2 cells. As shown in Fig. 2A and B, the results of RT-PCR and western blot analysis confirmed
Table II. Multivariate analysis of clinical variables.

\begin{tabular}{lll}
\hline & \multicolumn{2}{c}{ KLF9 expression } \\
\cline { 2 - 3 } $\begin{array}{l}\text { Clinicopathological } \\
\text { parameters }\end{array}$ & Relative risk (95\% CI) & P-value \\
\hline Sex (male) & $0.853(0.648-1.057)$ & 0.345 \\
Age (>50 years) & $0.564(0.429-0.699)$ & 0.157 \\
Differentiation & $0.775(0.589-0.961)$ & 0.642 \\
Lymphatic invasion & $0.654(0.497-0.811)$ & 0.332 \\
Venous invasion & $0.235(0.179-0.291)$ & 0.108 \\
Lymph node metastasis & $0.854(0.649-1.059)$ & 0.094 \\
Tumor size $(\geq 3$ cm) & $0.667(0.507-0.827)$ & 0.228 \\
KLF9 expression $(+-+++)$ & $1.235(0.939-1.531)$ & 0.043
\end{tabular}

CI, confidence interval. 
A
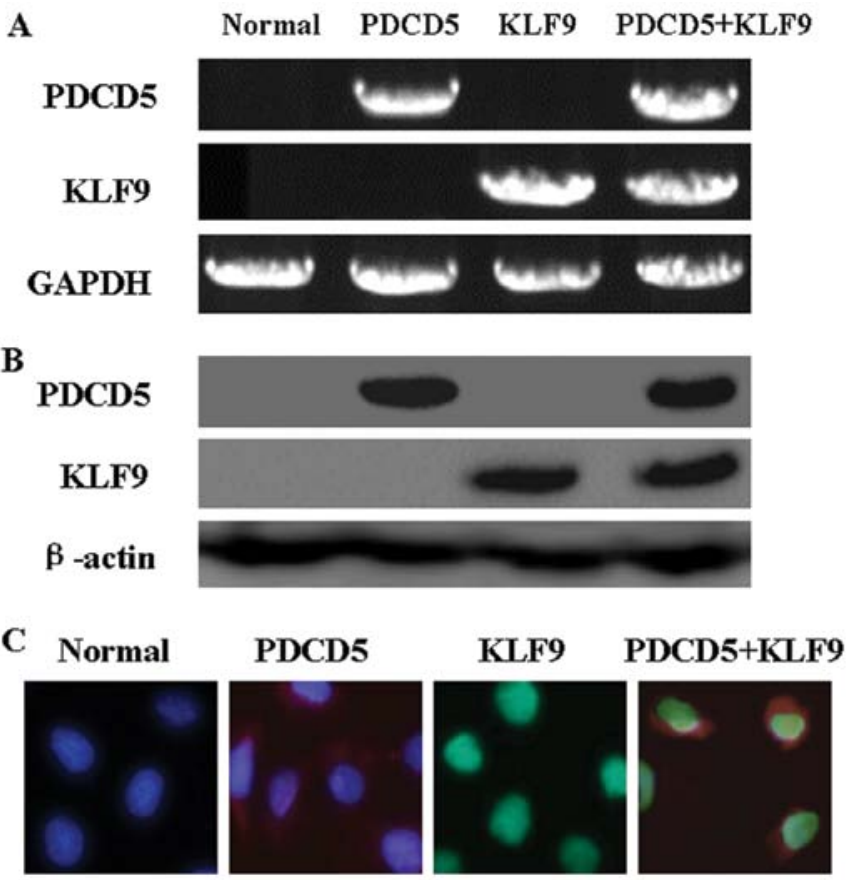

Figure 2. The levels of KLF9 and PDCD5 protein and mRNA were measured in HepG2 cells after transfection. (A) The mRNA levels of KLF9 and $P D C D 5$ were determined by RT-PCR. GAPDH was the internal control. (B) The expression of KLF9 and PDCD5 protein was determined by western blot analysis of HepG2 cells. $\beta$-actin expression was the internal control. (C) Detection of KLF9 and PDCD5 by immunofluorescence. exogenous expression of KLF9 and PDCD5 in HepG2 cells after transfection. Moreover, immunofluorescence analysis showed the localization of KLF9 and PDCD5 in transfected cells (Fig. 2C). The evidence confirmed that the co-transfection of KLF9 and PDCD5 in HepG2 cells was successful.

Growth inhibitory effects of KLF9 in PDCD5 expressing HepG2 cells. The proliferation rate of PDCD5-expressing HepG2 cells was significantly lower than untreated HepG2 cells by using a colony formation assay (Fig. 3A; $\mathrm{P}<0.05$ ). KLF9-expressing HepG2 cells showed no changes compared with untreated ones. Interesting, KLF9 inhibited the proliferation rate of PDCD5-expressing HepG2 cells (Fig. 3A; $\mathrm{P}<0.05$ ). Therefore, it is likely that PDCD5 expression would restore KLF9 antitumor activities in HepG2 cells. Condensed and fragmented nuclei and condensed chromatin was observed in co-expression of PDCD5 and KLF9 cells by using transmission electron microscopy (TEM) assay (Fig. 3B). The ratio of apoptotic cells in each group was determined by Annexin V and PI double-staining. PDCD5 and KLF9 expressing cells $(3.02 \pm 0.14 \%)$ exhibited an increased apoptosis compared to PDCD5 expressing ones $(2.24 \pm 0.08 \%)$, KLF9 expressing ones $(0.13 \pm 0.04 \%)$ and untreated ones $(0.17 \pm 0.03 \%)$ (Fig. 3C; $\mathrm{P}<0.05)$. As shown in Fig. 3D, the loss of membrane potential was indicated by the fluorescence emission shift from red to green. The ratio of red/green in the KLF9-expressing cells
$\mathbf{A}$
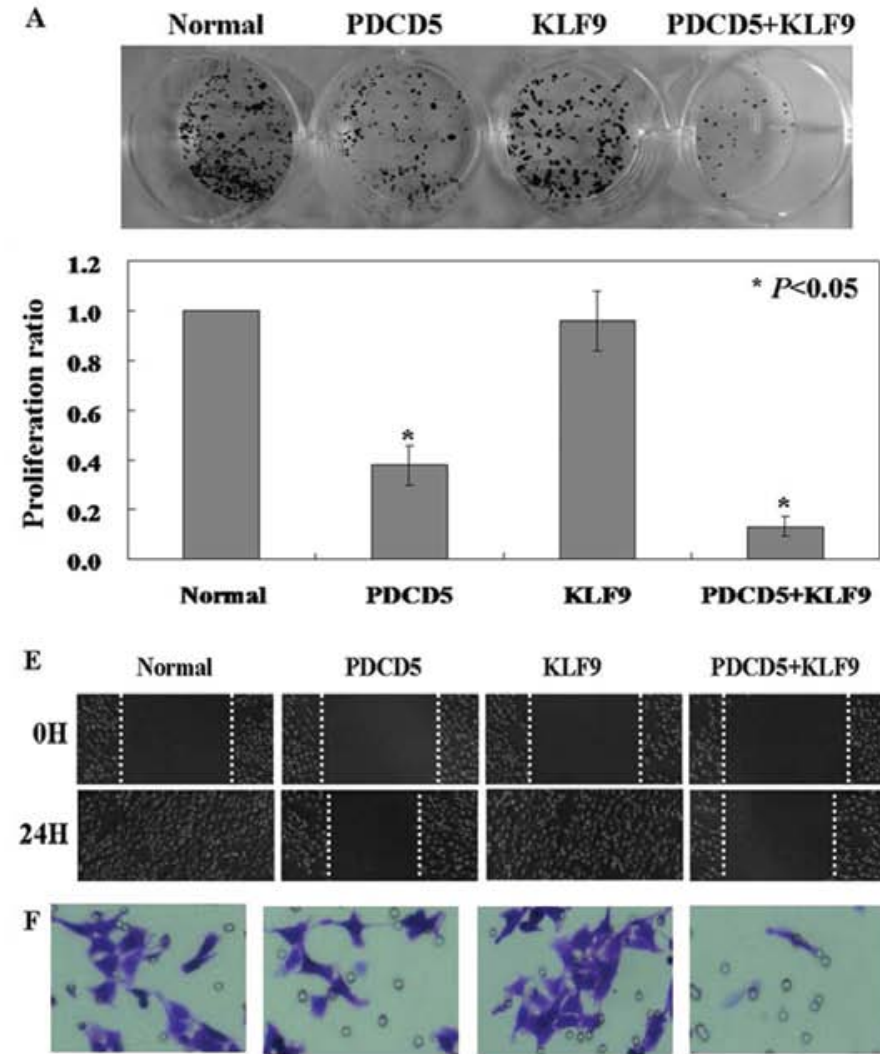
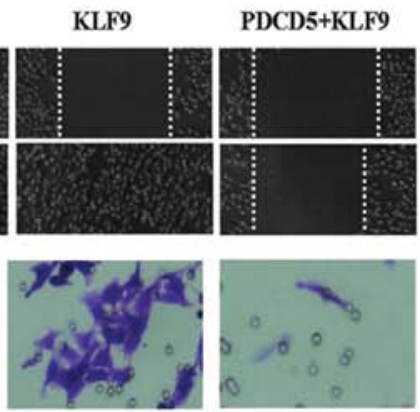

B
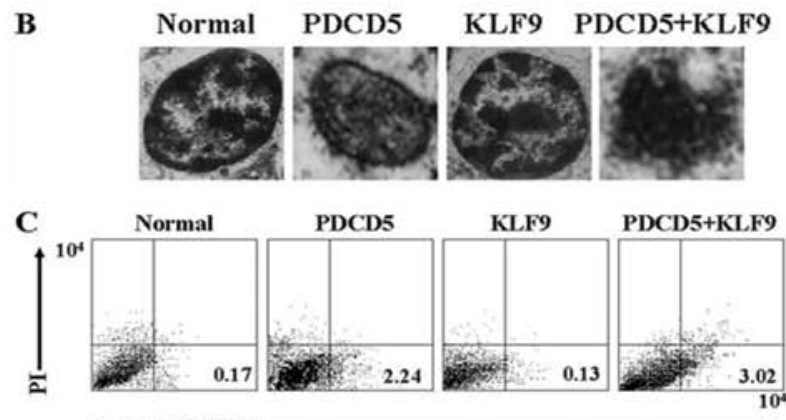

Annexin V-FITC
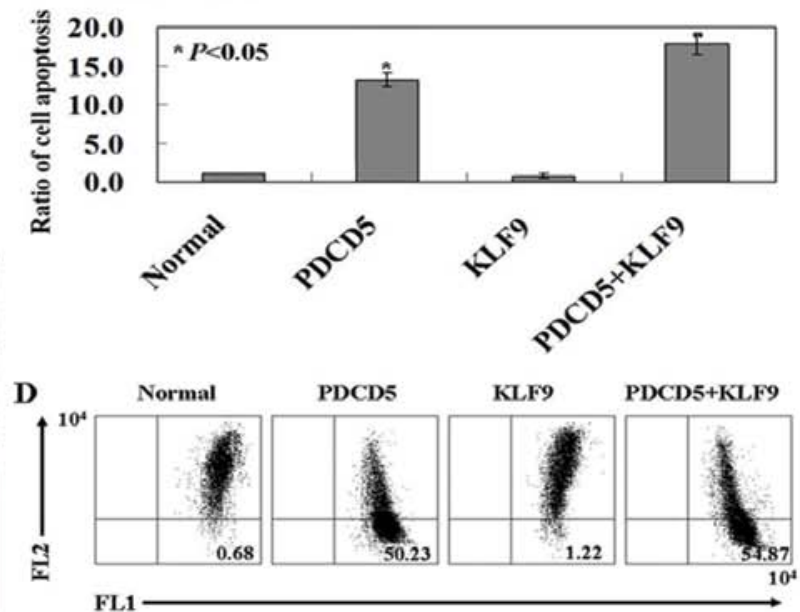

Figure 3. The antitumor activities of KLF9 and PDCD5 protein in HepG2 cells. (A) Colony formation assays were performed to determine the proliferation ratio of HepG2 cells. (B) TEM showed co-transfection cells with condensed and fragmented nuclei and condensed chromatin. (C) The proportion of apoptotic cells (early apoptosis) was determined by double-staining with Annexin-V/FITC and PI. (D) Mitochondrial-membrane potential was analyzed by flow cytometry. (E) Wound closure was quantified in the regions flanked by dotted lines. (F) Transwell assays were performed. Cells that migrated to the bottom side of the membrane were stained. Each experiment was performed in triplicate and was repeated three times. 


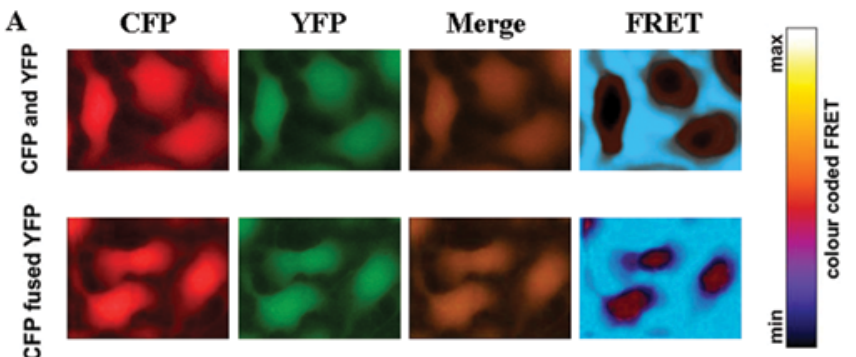

B

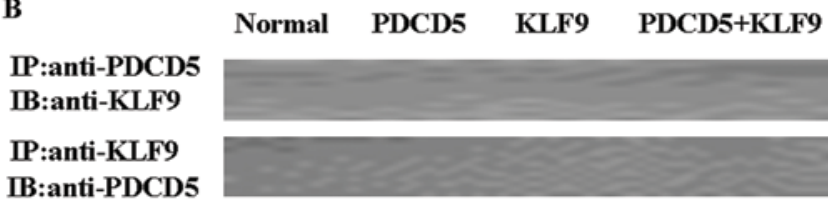

C

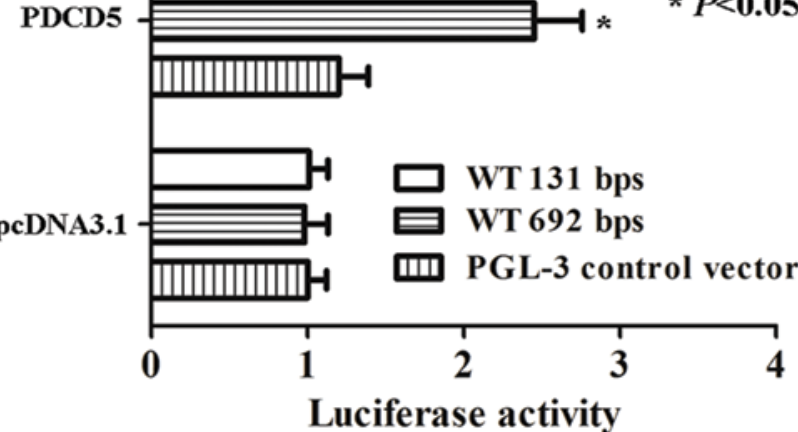

Figure 4. (A) Cells were grown on cover slips and cotransfected with CFP and YFP or the CFP-YFP fusion protein and mounted on microscope slides. Confocal images were taken and analyzed for FRET. (B) Protein extracts from co-transfection cells were immunoprecipitated with anti-KLF9 or anti-PDCD5 antibodies and immunoblotted with anti-PDCD5 or anti-KLF9 antibodies as indicated. (C) Lucifcrase activity was normalized to Renilla luciferase activity and then shown relative to that of pGL3-control-692-bp KLF9 promoter or pGL3-control-131-bp KLF9.
(1.22\% green) was reversed after co-transfected with PDCD5 (54.87\% green). The effect of KLF9 and PDCD5 expression on the motility of HepG2 cells was determined by wound-healing assay and transwell assay. The wound closure of the KLF9 and PDCD5 expressing cells was decreased when compared to the PDCD5 expressing ones, KLF9 expressing ones and untreated ones (Fig. 3E). The invasion of KLF9 and PDCD5 expressing cells was also significantly inhibited by comparing with the other three groups (Fig. 3F).

Analysis of interaction between PDCD5 and KLF9 by FRET, co-IP and luciferase assays. The interaction between CFP- and YFP-fused PDCD5 proteins is measured by the distance-dependent energy transfer from the excited donor CFP to the acceptor YFP. As shown in Fig. 4A, the FRET ratio (emission ratio 527:475 $\mathrm{nm}$ ) remained steady in KLF9 and PDCD5 expressing cells. The results indicated that PDCD5 protein had no interaction with KLF9 protein. Furthermore, we performed co-IP and western blot analysis. Consistent with the result of FRET, no interaction of PDCD5 and KLF9 was found (Fig. 4B). Interesting, in luciferase assays, expression driven by the 692-bp pGL3-control-KLF9 sequence was 2.3 times increased when PDCD5 was co-transfected in HepG2 cells. The smaller, 131-bp pGL3-control-KLF9 sequence resulted in an increase in luciferase expression induced by PDCD5 (Fig. 4C; $\mathrm{P}<0.05$ ).

Affymetrix GeneChip analysis. The expression of 3440 human transcripts was assessed using the Affymetrix ${ }^{\circledR}$ human expression array. We found that the expression of 236 genes was upregulated and the expression of 224 genes was downregulated in HepG2 cells with PDCD5 expression compared with untreated ones (Fig. 5; P<0.05). Upregulated expression of 121 genes and downregulated expression of

\section{Normal PDCD5 KLF9 PDCD5+KLF9}
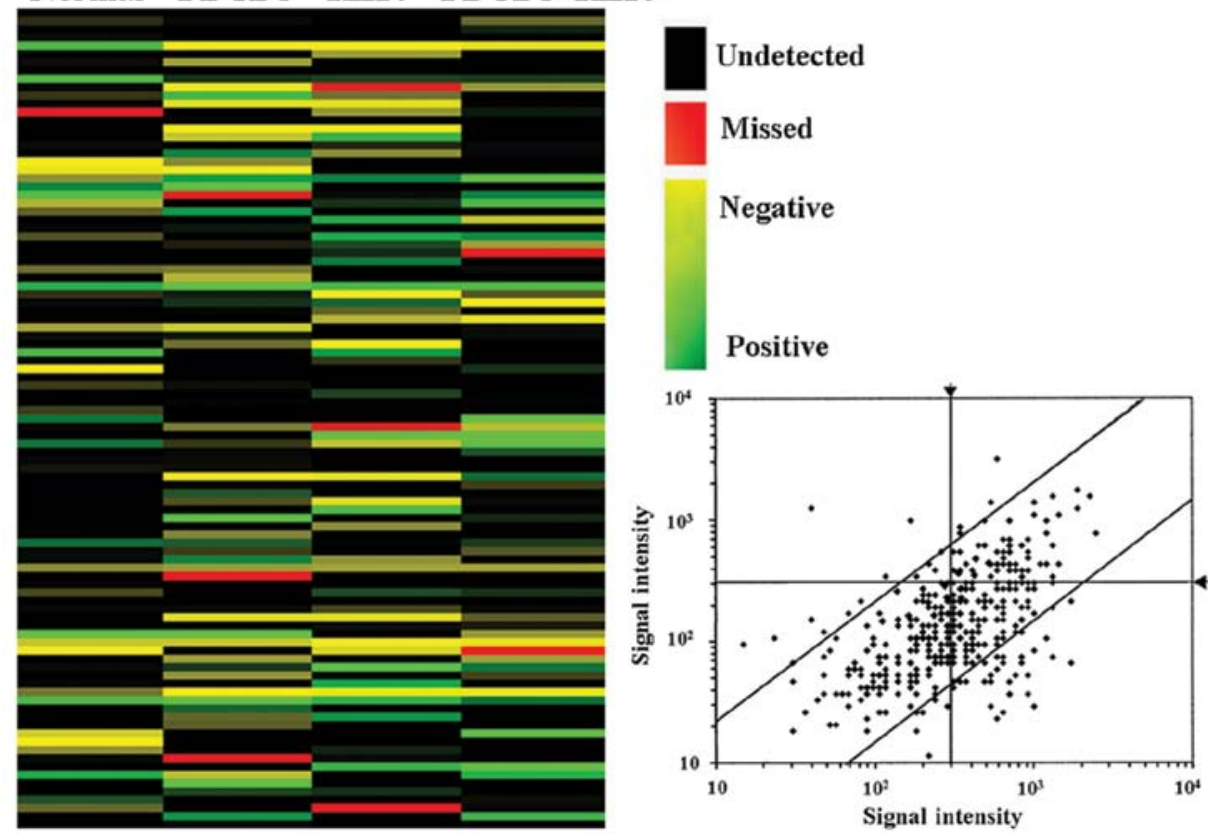

Figure 5. Microarray analysis identified many subtle differences in RNA expressions. The intensities are mean centered, with green indicating higher values and yellow indicating lower values. 


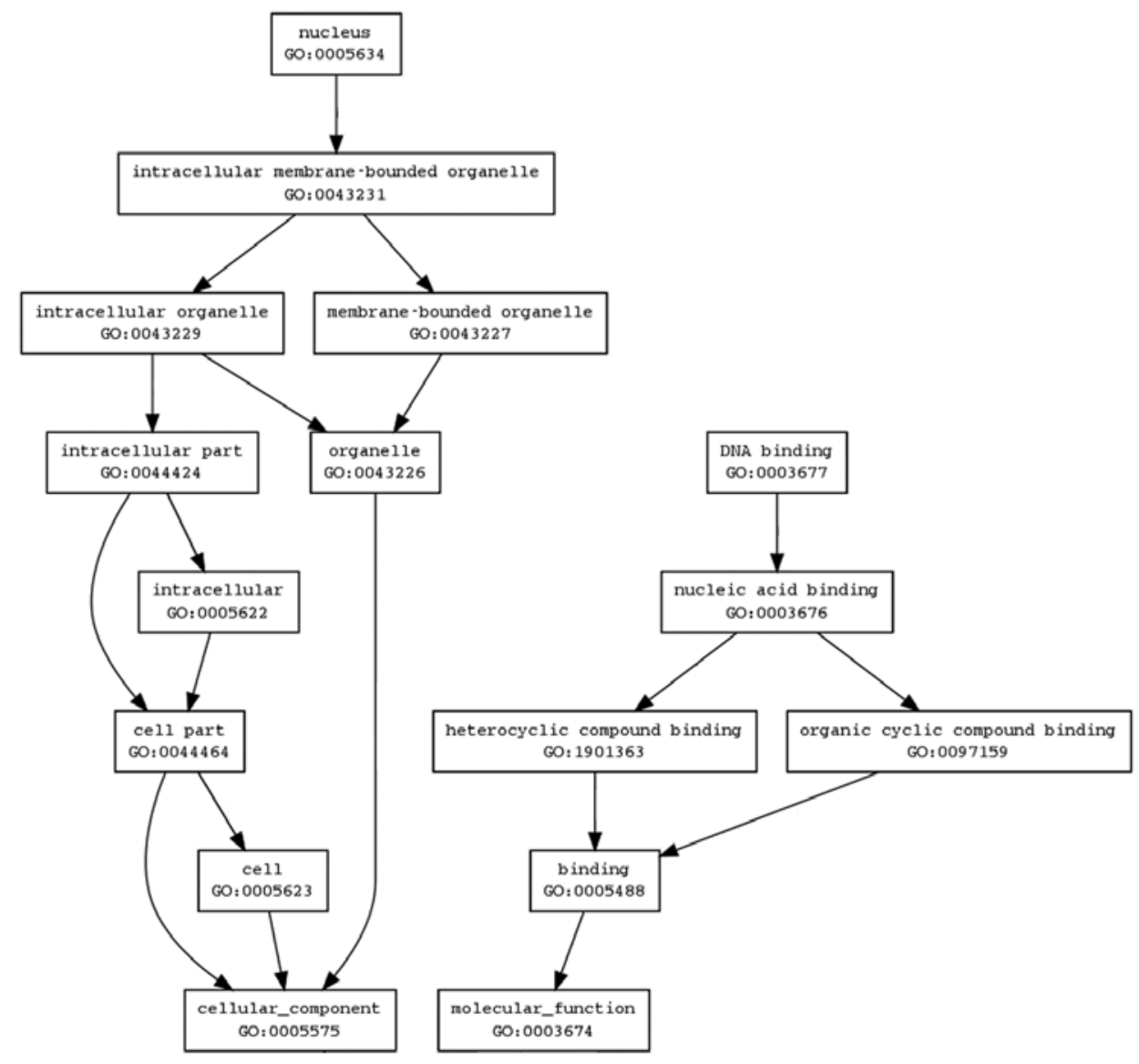

Figure 6. The canonical signaling pathways of PDCD5 were generated by using the Gene Ontology (GO) software (http://www.geneontology.org/).

287 genes were found in HepG2 cells with KLF9 expression compared with untreated ones (Fig. 5; P<0.05). Both PDCD5 and KLF9-induced upregulated expression of 253 genes and downregulated expression of 456 genes was observed in HepG2 cells (Fig. 5; P<0.05). We next investigated the biological interactions using the Bio MAS (molecule annotation system) 3.0 software and found the genes to map to genetic networks with functional relationships. The map is very complex and could not be elucidated in this study. Until now, we only arranged the network of PDCD5 and found the results were consistent with the results that were predicted by using the Gene Ontology (GO) software (http://www.geneontology.org/) (Fig. 6).

\section{Discussion}

The present study demonstrates for the first time that KLF9 mRNA and protein is downregulated in HCC samples, compared with matched normal tissues. Similar results were observed in the study of Kang et al (8) by detecting CRC tissues. Recent evidence suggests an association between KLF9 and human endometrial tumor pathology, with significantly higher KLF9 transcript levels in normal endometrium and stage I endometrial tumors when compared to more aggressive stage II-IV tumors (17). In our study, we confirmed that KLF9 expression was associated with the sex and lymph node metastasis of the patients.

One of the main findings of this study was that KLF9 played its role in HepG2 cells depending on PDCD5 expres- sion. Simmen et al (18) noted a regulatory role for KLF9 in crypt cell proliferation, villus cell migration, and Paneth and goblet cell differentiation in mice. However, we did not find antitumor activities of KLF9 in PDCD5-negative HepG2 cells. Therefore, we assumed that PDCD5 promotes KLF9-induced apoptosis in HepG2 cells. This hypothesis was demonstrated by using colony formation assay, Annexin V-FITC/PI double staining and transwell assay. PDCD5 is an apoptosis-promoting molecule that is upregulated in cells undergoing apoptosis $(19,20)$. Consistent with previous studies, in this study, we provide new evidence for PDCD5 as an apoptosis-promoting molecule.

Furthermore, we detected the possible mechanisms of KLF9 and PDCD5 in HepG2 cells. Although previous studies have reported the interaction of KLF1 and GATA1 in erythroid cells (21) and KLF13 and GATA4 in the heart (22), whether there is interaction between KLF9 and PDCD5 remains unclear. To study the protein interaction, we applied a non-invasive technique, FRET, in our study. Fluorescence (or Förster) resonance energy transfer (FRET) is based upon the transfer of energy from an excited donor fluorophor to a close-by acceptor fluorophor, resulting in enhanced fluorescence emission of the acceptor (23). We did not find any association of PDCD5 and KLF9. The result was confirmed by using co-IP assay. However, we demonstrated that the PDCD5-binding sites in the KLF9 promoter directly upregulate KLF9 expression. In the KLF9 promoter region, PDCD5-binding sites were clustered to 131-bp region and 692-bp region. 
We applied gene expression profiling studies to explore the changes of KLF9 and PDCD5 expressing cells. As noted in the Results, the network of KLF9 and PDCD5 is very complex. We will find the canonical signaling pathways that are influenced by KLF9 and PDCD5 and confirm these pathways by using corresponding inhibitors in our future studies.

\section{Acknowledgements}

We thank Dr Miao Yu for technical assistance.

\section{References}

1. Jemal A, Bray F, Center MM, et al: Global cancer statistics. CA Cancer J Clin 6: 69-90, 2011.

2. Bosch FX, Ribes J, Diaz M and Cléries R: Primary liver cancer: worldwide incidence and trends. Gastroenterology 127: S5-S16, 2004.

3. Ratziu V, Lalazar A, Wong L, et al: Zf9, a Kruppel-like transcription factor up-regulated in vivo during early hepatic fibrosis. Proc Natl Acad Sci USA 95: 9500-9505, 1998.

4. Wang N, Liu ZH, Ding F, Wang XQ, Zhou CN and Wu M: Down-regulation of gut-enriched Kruppel-like factor expression in esophageal cancer. World J Gastroenterol 8: 966-970, 2002.

5. Ghaleb AM, Aggarwal G, Bialkowska AB, Nandan MO and Yang VW: Notch inhibits expression of the Kruppel-like factor 4 tumor suppressor in the intestinal epithelium. Mol Cancer Res 6: 1920-1927, 2008.

6. Narla G, Heath KE, Reeves HL, et al: KLF6, a candidate tumor suppressor gene mutated in prostate cancer. Science 294 2563-2566, 2001.

7. Mukai S, Hiyama T, Tanaka S, Yoshihara M, Arihiro K and Chayama K: Involvement of Kruppel-like factor 6 (KLF6) mutation in the development of nonpolypoid colorectal carcinoma. World J Gastroenterol 13: 3932-3938, 2007.

8. Kang L, Lü B, Xu J, Hu H and Lai M: Downregulation of Krüppel-like factor 9 in human colorectal cancer. Pathol Int 58 : 334-338, 2008

9. Suske G, Bruford E and Philipsen S: Mammalian SP/KLF transcription factors: bring in the family. Genomics 85: 551-556, 2005.

10. Imataka H, Sogawa $\mathrm{K}$, Yasumoto $\mathrm{K}$, et al: Two regulatory proteins that bind to the basic transcription element (BTE), a GC box sequence in the promoter region of the rat P-4501 A1 gene. EMBO J 11: 3663-3671, 1992.
11. Ohe N, Yamasaki Y, Sogawa K, et al: Chromosomal localization and cDNA sequence of human BTEB, a GC box binding protein. Somat Cell Mol Genet 19: 499-503, 1993.

12. Jiang J, Chan YS, Loh YH, et al: A core Klf circuitry regulates self-renewal of embryonic stem cells. Nat Cell Biol 10: 353-360, 2008.

13. Nikolcheva T, Pyronnet S, Chou SY, Sonenberg N, Song A, Clayberger C and Krensky AM: A translational rheostat for RFLAT-1 regulates RANTES expression in T lymphocytes. J Clin Invest 110: 119-126, 2002.

14. Good KL and Tangye SG: Decreased expression of Kruppel-like factors in memory B cells induces the rapid response typical of secondary antibody responses. Proc Natl Acad Sci USA 104: 13420-13425, 2007.

15. Fu DZ, Cheng Y, He H, Liu HY and Liu YF: PDCD5 expression predicts a favorable outcome in patients with hepatocellular carcinoma. Int J Oncol 43: 821-830, 2013.

16. Du H, Sarno J and Taylor HS: HOXA10 inhibits Kruppel-like factor 9 expression in the human endometrial epithelium. Biol Reprod 83: 205-211, 2010

17. Simmen RC, Pabona JM, Velarde MC, et al: The emerging role of Kruppel-like factors in endocrine-responsive cancers of female reproductive tissues. J Endocrinol 204: 223-231, 2010

18. Simmen FA, Xiao R, Velarde MC, et al: Dysregulation of intestinal crypt cell proliferation and villus cell migration in mice lacking Krüppel-like factor 9. Am J Physiol Gastrointest Liver Physiol 292: G1757-G1769, 2007.

19. Wang Y, Li X, Wang L, et al: An alternative form of paraptosis like cell death, triggered by TAJ/TROYand enhanced by PDCD5 overexpression. J Cell Sci 117: 1525-1532, 2004.

20. Chen Y, Sun R, Han W, et al: Nuclear translocation of PDCD5 (TFAR19): an early signal for apoptosis? FEBS Lett 509: 191-196, 2001.

21. Cantor $\mathrm{AB}$ and Orkin $\mathrm{SH}$ : Transcriptional regulation of erythropoiesis: an affair involving multiple partners. Oncogene 21: 3368-3376, 2002.

22. Lavallée G, Andelfinger G, Nadeau M, Lefebvre C, Nemer G, Horb ME and Nemer M: The Kruppel-like transcription factor KLF13 is a novel regulator of heart development. EMBO J 25: 5201-5213, 2006.

23. Selvin PR: The renaissance of fluorescence resonance energy transfer. Nat Struct Biol 7: 730-734, 2000. 